\title{
CANONICAL BASES IN EXCELLENT CLASSES
}

\author{
TAPANI HYTTINEN AND OLIVIER LESSMANN
}

\begin{abstract}
We show that any (atomic) excellent class $\mathscr{K}$ can be expanded with hyperimaginaries to form an (atomic) excellent class $\mathscr{K}^{e q}$ which has canonical bases. When $\mathscr{K}$ is, in addition, of finite $U$-rank, then $\mathscr{K}^{\text {eq }}$ is also simple and has a full canonical bases theorem. This positive situation contrasts starkly with homogeneous model theory for example, where the eq-expansion may fail to be homogeneous. However. this paper shows that expanding an $\omega$-stable, homogeneous class $\mathscr{H}$ gives rise to an excellent class, which is simple if $\mathscr{H}$ is of finite $U$-rank.
\end{abstract}

Introduction. One of the central tools of geometric stability theory is that we may assume that the monster model $\mathfrak{C}$ has canonical bases i.e., for each complete type $p$ over $\mathfrak{C}$ there is an element $C b(p) \in \mathfrak{C}$ with the property that automorphisms of $\mathfrak{C}$ fix $p$ if and only if they fix $C b(p)$. Further, this canonical basis behaves extremely well with respect to the independence relation given by nonforking; this is the content of Shelah's Canonical Basis Theorem: For $p$ a complete type over $\mathfrak{C}$ and $A$ a subset of $\mathfrak{C}$ we have that $p$ does not fork over $A$ if and only if $C b(p)$ is in the algebraic closure of $A$, and $p\lceil A$ is, in addition, stationary if and only if $C b(p)$ is in the definable closure of $A$.

Obtaining canonical bases in a stable first order theory is achieved by expanding the language of the original theory with a new sort $S_{E}$ for each definable equivalence relation $E$, which is then interpreted as the set of equivalence classes $a / E$, for $a \in \mathfrak{C}$. These new elements are called imaginaries. The crucial point is that, not only does this expansion provide the necessary canonical bases, but it also preserves all the important features; completeness, saturation, categoricity, stability, and so forth. Trying to do this for simple, rather than stable, first order theories is still possible (as shown by Bradd Hart, Bynghan Kim, and Anand Pillay in [HKP]) but more delicate, as one is lead to consider type-definable equivalence relations (the added elements are called hyper-imaginaries). In particular, the expansion is no longer first order (but homogeneous and manageable).

Another motivation is that groups appearing in geometric model theory are often only interpretable (that is, they are obtained as a quotient of a definable set by a definable equivalence relation). Expanding with imaginaries allows one to assume that they are definable. Then, the whole first order theory of stable groups apply and a lot of information is known on such groups.

The goal of this paper is to find such a well-behaved expansion in the context of excellent classes. Excellence is a property originally defined by S. Shelah (see [Sh87a]

Received August 12, 2006. 
and [Sh87b] in his work around categoricity for nonelementary classes which has found applications in Boris Zilber's work on complex exponetiation (see [Zi]). Groups in the context of excellent classes have been studied in [HLS]. We also examine the particular case when the class we start with has finite $U$-rank. This is a natural assumption as all the classes studied by Zilber have finite $U$-rank, and it is shown in [HyLe] that uncountably categorical classes are essentially of finite $U$-rank.

In this paper, we fix an excellent atomic class $\mathscr{K}$ in a countable language $L$ and work in the full model $\mathfrak{C}$.

We consider the set $T E(\mathscr{K})$ of type-definable equivalence relations on the models of $\mathscr{K}$, so we will expand our structures with hyper-imaginaries. A basic technical problem is that we would like to keep the new language countable (to preserve the existence of countable models and $\omega$-stability), so we fix a countable subset $\mathscr{E}$ of $T E(\mathscr{K})$. We show that if we expand the language $L$ to a language $L_{\mathscr{E}}$ containing a new sort for each equivalence relation $E \in \mathscr{E}$, interpreted in $\mathfrak{C}$ as the set of equivalence classes $a / E$, and a new function symbol $F_{E}$, interpreted as $F_{E}(a)=a / E$, then the resulting class $\mathscr{K}^{\mathscr{E}}$ is atomic and excellent. This expansion also preserves the categoricity spectrum and furthermore, if $\mathscr{K}$ is of finite $U$-rank (and hence simple, see [HyLe]), then $\mathscr{K}^{\mathscr{E}}$ is simple.

This contrasts already starkly with the homogeneous case: Even if $\mathscr{K}$ is homogeneous and $\omega$-stable, $\mathscr{K}^{\mathscr{E}}$ may fail to be homogeneous. However, our result shows that $\mathscr{K}^{\mathscr{\mathscr { C }}}$ is still excellent. Furthermore, without stability, even if $\mathscr{K}$ was simple, then $\mathscr{K}^{\mathscr{E}}$ can fail to be simple (see [BY]). One of the reasons why this works here is that the $\omega$-stability of $\mathscr{K}$ implies that we have a good behaviour of indiscernibles in the hyper-imaginaries.

In the second part of the paper, we show that we can choose a countable set $\mathscr{E}$ such that each complete type over $\mathfrak{C}^{\mathscr{E}} \in \mathscr{K}^{\mathscr{E}}$ has a canonical basis and satisfies the Canonical Basis Theorem; we call the resulting class $\mathscr{K}^{e q}$ and its models $M^{e q}$. We do this at two levels of generality: Under the assumption that $\mathscr{K}$ is excellent (in this case we work with splitting and types over models) and under the assumption that $\mathscr{K}$ is excellent and of finite $U$-rank (in this we we work with Lascar-splitting and types over all sets). In this paper, we prove two main theorems. They are versions of the Canonical Basis Theorem at two levels of generality. In the context of excellence:

The main theorem of the paper is the Canonical Basis Theorem:

THEOREM. Let $\mathscr{K}$ be an excellent, atomic class in the countable language $L$. Let $\mathfrak{C} \in \mathscr{K}$ be full. Then $\mathscr{K}^{e q}$ is excellent and atomic in the countable language $L^{e q}$, and $\mathfrak{C}^{e q} \in \mathscr{K}^{e q}$ is full. Also, for each complete $p$ over $\mathfrak{C}^{e q}$, there exists an element $C b(p) \in \mathfrak{C}^{e q}$ with the property that

$$
f(p)=p \quad \text { if and only if } \quad f(C b(p))=C b(p), \quad \text { for each } f \in \operatorname{Aut}\left(\mathfrak{C}^{e q}\right) .
$$

The element $C b(p)$ in the previous theorem is called the em canonical basis of $p$. We can prove a better theorem if we assume, in addition, that $\mathscr{K}$ has finite $U$-rank:

THEOREM. Let $\mathscr{K}$ be an excellent, atomic class in the countable language $L$ and of finite $U$-rank. Let $\mathfrak{C} \in \mathscr{K}$ be full. Then $\mathscr{K}^{e q}$ is simple, excellent, atomic in the countable language $L^{e q}$, and $\mathfrak{C}^{e q} \in \mathscr{K}^{e q}$ is full. Moreover, for each complete type $p$ over $\mathfrak{C}^{e q}$, the canonical basis $C b(p)$ has the following two properties: 
(1) $p$ is an independent extension of $p\left\lceil A\right.$ if and only if $C b(p) \in b c l^{e q}(A)$,

(2) if $A$ is finite, then $p$ is the only independent extension of the stationary $p\lceil A$ if and only if $C b(p) \in d c l^{e q}(A)$.

The key tool is type-definability of types over models (initially shown by Shelah in [Sh48]) and stationarity of Lascar strong types (shown by the authors in [HyLe]).

The paper is organised as follows. The first section contains basic results on expanding an atomic class with invariant equivalence relations. The second section is concerned with the excellent case and contains the first canonical basis theorem above. The last section is concerned with the case when the initial class is of finite $U$-rank. For all relevant preliminaries on the basics of excellence the reader is invited to consult [Le2] or [HyLe] where simplicity is introduced at this level of generality.

We finish this introduction by giving a very simple example of objects studied in this paper. Let $\mathscr{K}$ be the class of all models $M$ as follows: $M$ interprets two binary predicates $E$ and $F$ so that the interpretations are equivalence relations with infinitely many equivalence classes, $F$ is finer than $E$ and each $E$-equivalence class is split by $F$ into countably many $F$-equivalence classes so that for all non-zero natural numbers $n$ there is exactly one class of size $n$. Then $\mathscr{R}$ is an atomic totally categorical excellent class (even homogeneous), it is $\omega$-stable and of finite $U$-rank (even quasi-minimal) and (thus) simple. Now, for all sets $X$ of natural numbers one can define a type-definable equivalence relation $E_{X}$ as follows: $(a, b) \in E_{X}$ if $E(a, b)$ holds and the sizes of $a / F$ and $b / F$ are either both in $X$ or neither is in $X$. A straigth forward Ehrenfeucht-Fraisse-game argument shows that if $X$ and it's complement are infinite then $E_{X}$ is not definable.

§0. Expanding with hyperimaginaries. In this section, we show that expanding naturally an atomic class with countably many type-definable equivalence relations gives rise to an atomic class with similar properties.

Let $\mathscr{K}$ be the class of atomic models of a complete first order theory $T$ in the countable language $L$. We make a few observations concerning type-definable (over the empty set) equivalence relations on the models of $\mathscr{K}$.

Suppose that $E(x, y)$ is a type-definable relation and let $M \prec N \in \mathscr{K}$. If $E(x, y)$ is an equivalence relation on $N$, then clearly it is an equivalence relation on $M$, but the converse holds also: If $E(x, y)$ fails to be reflexive on $N$, then for some $a \in N$ and some formula $\phi(x, y) \in E$ we have $N \models \neg \phi(a, a)$. Let $a^{\prime} \in M$ realise $\operatorname{tp}(a / \emptyset)$ ( $a^{\prime}$ exists since $N$ is atomic). Then $M \models \neg \phi\left(a^{\prime}, a^{\prime}\right)$ so $E$ is not reflexive on $M$. If $E(x, y)$ is not symmetric on $N$, then for some $a, b \in N$ realising $E(x, y)$ there is $\phi(x, y) \in E$ such that $N \models \neg \phi(b, a)$. Again, let $a^{\prime} b^{\prime} \in M$ realise $\operatorname{tp}(a b / \emptyset)$. Then $a^{\prime} b^{\prime}$ realise $E(x, y)$ but $b^{\prime} a^{\prime}$ does not realise $E(x, y)$, so $E$ is not symmetric on $M$. The transitivity is similar: If $a, b, c \in N$ are such that $E(a, b), E(b, c)$ but $a, c$ do not realise $E(x, y)$, then $a^{\prime} b^{\prime} c^{\prime} \in M$ realising $\operatorname{tp}(a b c / \emptyset)$ witness the failure of the transitivity of $E$ on $M$. Thus, a type $E(x, y)$ defines an equivalence relation on one model of $\mathscr{K}$ if and only if it defines an equivalence relation on all models of $\mathscr{K}$.

Further, if $E_{1}, E_{2}$ are type definable equivalence relations which are distinct on $N$ and $M \prec N$, then $E_{1}$ and $E_{2}$ are distinct on $M$ : if $(a, b) \in N$ realise $E_{1}$ but not $E_{2}$, then $\left(a^{\prime}, b^{\prime}\right) \in M$ realising $\operatorname{tp}(a b / \emptyset)$ realise $E_{1}$ but not $E_{2}$. 
Finally, if $M \in K$ is strongly $\omega$-homogeneous (for example when $M$ is countable), then any subset of $M$ is invariant if and only if it is type-definable (as $M$ is also atomic): Certainly, if $X$ is type-definable, then $X$ is invariant in $M$. For the converse, suppose that $X$ is invariant. Let $S_{X}=\{\operatorname{tp}(a / \emptyset): a \in M \backslash X\}$ and let $\phi_{p} \models p$ for each $p \in S_{X}$. Then, by invariance of $X$ and strong $\omega$-homogeneity of $M, a \in X$ if and only if $a \models \bigwedge\left\{\neg \phi_{p}: p \in S_{X}\right\}$, so $X$ is type-definable.

In order to simplify notation, we assume that the models in $\mathscr{K}$ are one-sorted. However, our expansions are many sorted and we want to iterate the expansion construction and so we give definitions in a form which allows models in $\mathscr{K}$ to be many sorted but we prove everything under the assumption that the models are one-sorted. From this, it is easy to see that everything holds also in the case when the models in $\mathscr{K}$ are many sorted. By $S(M)$ we denote the set of all elements of $M$ of sort $S$.

Definition 0.1 . We let $T E(\mathscr{K})$ to be the collection of type-definable equivalence relations $E$ on the models $M$ of $\mathscr{K}$ such that

$$
E \subseteq\left(S_{0}(M) \times \cdots \times S_{n}(M)\right) \times\left(S_{0}(M) \times \cdots \times S_{n}(M)\right)
$$

for some sorts $S_{0}, \ldots, S_{n}$.

The remarks above the definition show that $T E(\mathscr{K})$ corresponds to the invariant equivalence relations on the unique countable model of $\mathscr{K}$.

We would like to expand the language $L$ with a new sort for each $E \in T E(\mathscr{K})$. Unfortunately, the set $T E(\mathscr{K})$ may have size continuum, so the size of the expanded language would change. This would immediately prevent the existence of countable models in the expanded class, and destroy $\omega$-stability and $\aleph_{0}$-categoricity. Thus, we always consider countable subsets of $T E(\mathscr{K})$ (containing $=$ ) to form our expansions. We will see later in this paper that considering countable expansions is enough. In practice, it will always be convenient to assume closedness under finitary operations, like finite intersections or cartesian products, but it is not necessary here.

For the rest of this section, let $\mathscr{E} \subseteq T E(\mathscr{K})$ be countable containing the equality for each sort $S$ of the models of $\mathscr{K}$, these relations are denoted by $=(S)$. For each $E \in \mathscr{E}$, the language $L^{\mathscr{E}}$ will contain a new sort $S_{E}$ and a new function symbol $F_{E}$ from the appropriate sort $S_{=\left(S_{0}\right)} \times \cdots \times S_{=\left(S_{n}\right)}$ into the sort $S_{E}$. For $M \in \mathscr{K}$, the model $M^{\mathscr{E}}$ is the natural expansion of $M$ in $L^{\mathscr{E}}$ where the sort $S_{E}$ is interpreted as $\left\{a / E: a \in S_{0}(M) \times \cdots \times S_{n}(M)\right\}$ with identification of $S_{=\left(S_{i}\right)}\left(M^{\mathscr{E}}\right)$ with $S_{i}(M)$ and we let $F_{E}(a)=a / E$, with $a \in S_{0}(M) \times \cdots \times S_{n}(M)$. In keeping with the name used for them in first order simplicity theory [HKP], we call hyperimaginaries the elements $a / E \in M^{\mathscr{E}}$.

If $M^{\mathscr{E}} \prec N^{\mathscr{E}}$ then $M \prec N$, and more generally if $g: M^{\mathscr{E}} \rightarrow N^{\mathscr{E}}$ is elementary in the language $L^{\mathscr{E}}$, then the restriction to $M$ is elementary (in $L$ ) into $N$. If $M \prec N$, then not necessarily $M^{\mathscr{E}} \prec N^{\mathscr{E}}$, as there is some arbitrariness in forming the expansion. However, the following holds:

Proposition 0.2. Let $f: M \rightarrow N$ be an elementary map. Then there exists a unique $L^{\mathscr{E}}$-elementary map $f^{\mathscr{E}}: M^{\mathscr{E}} \rightarrow N^{\mathscr{E}}$. Further, if $f: M \rightarrow N$ is an isomorphism, then $f^{\mathscr{E}}: M^{\mathscr{E}} \rightarrow N^{\mathscr{E}}$ is an isomorphism. 
Proof. We define $f^{\mathscr{E}}(a / E):=f(a) / E$, for each $a / E \in M^{\mathscr{E}}$ and $E \in \mathscr{E}$. This is well-defined: If $a / E=b / E$ in $M^{\mathscr{E}}$ then $E(a, b)$ in $M$, so $E(f(a), f(b))$ in $N$ so $N^{\mathscr{E}} \models f(a) / E=f(b) / E$. It is now easy to show that $f^{\mathscr{E}}$ is elementary. The uniqueness is clear so is the last sentence.

So starting from the same model $M$, all the $\mathscr{E}$-expansions are isomorphic; this is why, when it does not matter, we may simply say $M^{\mathscr{E}}$, as though it were uniquely defined. The following consequence will be used repeatedly:

Proposition 0.3. Let $M^{\mathscr{E}} \prec N^{\mathscr{E}}$ and let $f$ be an automorphism of $N^{\mathscr{E}}$ fixing $M$ (i.e., $S_{=}\left(M^{\mathscr{E}}\right)$ ). Then $f$ fixes $M^{\mathscr{E}}$.

Proof. The restriction of $f$ to $M^{\mathscr{E}}$ is an elementary map into $N^{\mathscr{E}}$ which coincides with id : $M^{\mathscr{E}} \rightarrow N^{\mathscr{E}}$ (also elementary) on $M$. Thus $f$ is the identity on $M^{\mathscr{E}}$ by uniqueness (Proposition 0.2).

REMARK 0.4 . Note that if $E(x, y) \in T E(\mathscr{K})$ and $E^{\prime}\left(x x^{\prime}, y y^{\prime}\right)$ is defined by $E(x, y)$ (that is, we add dummy variables), then $E^{\prime}\left(x x^{\prime}, y y^{\prime}\right) \in T E(\mathscr{K})$. Also, if $E_{1}(x, y)$ and $E_{2}(x, y) \in T E(\mathscr{K})$ and $E(x, y)$ holds if and only if $E_{1}(x, y)$ and $E_{2}(x, y)$ both hold, then $E(x, y) \in T E(\mathscr{K})$. So, by closing $\mathscr{E}$ under dummy variables and finite intersections, we can reduce tuples of elements to elements: If $a_{i} / E_{i}$ are given, for $i<n$, with $E_{i}\left(x_{i}, y_{i}\right) \in \mathscr{E}$, we can consider

$$
E_{i}^{\prime}\left(x_{1} \ldots x_{n-1}, y_{1} \ldots y_{n-1}\right) \in \mathscr{E}
$$

defined by $E_{i}\left(x_{i}, y_{i}\right)$ and then

$$
E\left(x_{1} \ldots x_{n-1}, y_{1} \ldots y_{n-1}\right) \in \mathscr{E}
$$

given by the intersection of $E_{i}^{\prime}$, for $i<n$. Then the tuple $a_{0} / E_{0}, \ldots, a_{n_{1}} / E_{n-1} \in M^{\mathscr{E}}$ is interdefinable with the element $\left(a_{0}, \ldots, a_{n-1}\right) / E$.

We now consider homogeneity. Recall that a model $M$ is strongly $\lambda$-homogeneous, for a cardinal $\lambda$, if whenever $a, b \in M$ are sequences of length less than $\lambda$ and $\operatorname{tp}_{L}(a / \emptyset)=\operatorname{tp}_{L}(b / \emptyset)$ then there exists an automorphism $f$ of $M$ such that $f(a)=b$. Observe that since any $A \subseteq N^{\mathscr{E}}$ is in the $L^{\mathscr{E}}$-definable closure (in the first order sense) of some $A^{\prime} \subseteq N$, and if $f$ is an automorphism of $N$ fixing $A^{\prime}$ and sending $a$ to $b$. Then $f^{\mathscr{E}}$ is an automorphism of $N^{\mathscr{E}}$ fixing $A$ sending $a$ to $b$. However, this does not imply that $N^{\mathscr{E}}$ is strongly $\lambda$-homogeneous if $N$ is, as we need to move elements which may not be in $S_{=}\left(N^{\mathscr{E}}\right)$. In general actually, $\lambda$-strong homogeneity is not preserved for uncountable $\lambda$, but it is for $\lambda=\omega$ :

Proposition 0.5. Let $M \in \mathscr{K}$ be strongly $\omega$-homogeneous. Then $M^{\mathscr{E}}$ is strongly $\omega$-homogeneous (in $L^{\mathscr{E}}$ ).

Proof. We must show that if

$$
\operatorname{tp}_{L^{\mathscr{E}}}\left(a_{0} / E_{0}, \ldots, a_{n-1} / E_{n-1} / \emptyset\right)=\operatorname{tp}_{L^{\mathscr{E}}}\left(b_{0} / E_{0}, \ldots, b_{n-1} / E_{n-1} / \emptyset\right),
$$

then there is an automorphism of $M^{\mathscr{E}}$ sending each $a_{i} / E_{i}$ to $b_{i} / E_{i}$, for $i<n$.

Let $\phi\left(x_{0}, \ldots, x_{n-1}\right) \in L^{\mathscr{E}}$ be the formula

$$
\exists z_{0} \ldots \exists z_{n-1}\left(\bigwedge_{i<n} x_{i}=F_{E_{i}}\left(z_{i}\right) \wedge \psi\left(z_{0}, \ldots, z_{n-1}\right)\right),
$$

where $\psi$ isolates the type $\operatorname{tp}_{L}\left(a_{0}, \ldots, a_{n-1} / \emptyset\right)$. Then since

$$
\phi \in \operatorname{tp}_{L^{\varepsilon}}\left(a_{0} / E_{0}, \ldots, a_{n-1} / E_{n-1} / \emptyset\right),
$$


by $(*)$ there are $b_{0}^{\prime}, \ldots, b_{n-1}^{\prime} \in M$ realising $\operatorname{tp}_{L}\left(a_{0}, \ldots, a_{n-1} / \emptyset\right)$, such that $M^{\mathscr{E}} \models$ $b_{i} / E_{i}=b_{i}^{\prime} / E_{i}$, for $i<n$. By strong $\omega$-homogeneity of $M$, there is an automorphism $f$ of $M$ such that $f\left(a_{i}\right)=b_{i}^{\prime}$ for $i<n$. By Proposition 0.2 , this automorphism $f$ extends uniquely to an automorphism $f^{\mathscr{E}}$ of $M^{\mathscr{E}}$ which sends $a_{i} / E_{i}$ to $b_{i}^{\prime} / E_{i}$ and hence $b_{i} / E_{i}$, for $i<n$.

Proposition 0.6. The models $M^{\mathscr{E}}$ are atomic (in $L^{\mathscr{E}}$ ) for each $M \in \mathscr{K}$.

Proof. Let $a_{i} / E_{i} \in M^{\mathscr{E}}$ for $i<n$ be given. We must show that the type

$$
\operatorname{tp}_{L^{\mathscr{E}}}\left(a_{0} / E_{0}, \ldots, a_{n-1} / E_{n-1} / \emptyset\right)
$$

is isolated. Let $\phi\left(x_{0}, \ldots, x_{n-1}\right)$ be as in the previous proof. Let $b_{i} / E_{i}$ for $i<n$ with $M^{\mathscr{E}} \models \phi\left(b_{0} / E_{0}, \ldots, b_{n-1} / E_{n-1}\right)$. We may assume that $M$ is countable, so like the previous proof there is an automorphism of $M^{\mathscr{E}}$ sending $b_{i} / E_{i}$ to $a_{i} / E_{i}$, which proves that $\phi \models \operatorname{tp}_{L^{\mathscr{E}}}\left(a_{0} / E_{0}, \ldots, a_{n-1} / E_{n-1} / \emptyset\right)$.

Definition 0.7. Let $\mathscr{K}^{\mathscr{E}}=\left\{M^{\mathscr{E}}: M \in \mathscr{K}\right\}$.

The next proposition is an easy consequence of the work we have done:

Proposition 0.8. The class $\mathscr{K}^{\mathscr{E}}$ consists of the atomic models in the countable language $L^{\mathscr{E}}$ of the first order theory $T^{\mathscr{E}}=T h_{L^{\mathscr{E}}}\left(M^{\mathscr{E}}\right)$, where $M$ is the countable model of $\mathscr{K}$.

Furthermore, we have also shown that:

Proposition 0.9. (1) If $\mathscr{K}$ has the amalgamation property then $\mathscr{K}^{\mathscr{E}}$ has the amalgamation property.

(2) If $\mathscr{K}$ has the joint embedding property then $\mathscr{K}^{\mathscr{B}}$ has the joint embedding property.

(3) If $\mathscr{K}$ is categorical in some cardinal $\lambda$, then $\mathscr{K}^{\mathscr{E}}$ is categorical in $\lambda$.

The first item is significant here: Even though AP holds, SAP, that is the Set Amalgamation Property, can fail for $\mathscr{K}^{\mathscr{E}}$ even though it holds for $\mathscr{K}$ (this is the same as saying that homogeneity is not preserved). The third item uses the countability of $\mathscr{E}$.

In principle, there could now be essentially new type-definable equivalence relation (in the language $L^{\mathscr{E}}$ ). This, however, is not the case:

Proposition 0.10. If $E$ is an $L^{\mathscr{E}}$-type-definable equivalence relation on $S_{=}\left(M^{\mathscr{E}}\right)^{n}$, then $E \in T E(\mathscr{K})$.

Proof. Since $\mathscr{K}^{\mathscr{E}}$ is atomic, we may assume that $M$ is countable. (see the remark following Definition 0.1).

So, let $E(x, y)$ by $L^{\mathscr{E}}$-type definable on $\{a /=: a \in M\}$. Then $E(x, y)$ is invariant on $M$ (in the language $L$ ): If $f \in A u t(M)$ and $E(a, b)$, then $\operatorname{tp}_{L}(a b / \emptyset)=$ $\operatorname{tp}_{L}(f(a) f(b) / \emptyset)$, which implies that $\operatorname{tp}_{L^{E}}(a b / \emptyset)=\operatorname{tp}_{L^{E}}(f(a) f(b) / \emptyset)$ (since $f$ extends to an automorphism $f^{\mathscr{E}}$ of $M^{\mathscr{E}}$ which agrees with $f$ on $M$ ). Hence, $E(f(a), f(b))$, since $E(x, y) \subseteq \operatorname{tp}_{L^{8}}(a, b / \emptyset)$, so $E$ is invariant on $M$. But invariance in $M$ implies type-definable in $L$, since $M$ is strongly $\omega$-homogeneous.

$\S 1$. Preserving excellence and simplicity. Throughout this section, we assume that the atomic class $\mathscr{K}$ in the countable language $L$ is also excellent i.e., $\mathscr{K}$ is $\omega$-stable (see Definition 1.3) and satisfies Definition 1.6 below (recall that the reader is invited 
to consult [Le2] for the basic properties of excellent classes). We fix $\mathfrak{C}$ a large full model of $\mathscr{K}$ and work inside $\mathfrak{C}$ (i.e., $\mathfrak{C}$ is the monster model).

We fix a countable $\mathscr{E} \subseteq \mathscr{K}$ containing $=$, and we may assume that $\mathscr{E}$ is closed under dummy variables and finite intersections. We will show in a series of propositions that $\mathscr{K}^{\mathscr{E}}$ is also excellent, and further, if $\mathscr{K}$ is excellent and of finite $U$-rank, then $\mathscr{K}^{\mathscr{E}}$ is excellent and simple.

We have already established that $\mathscr{K}^{\mathscr{E}}$ is the class of atomic models of a countable first order theory $T^{\mathscr{E}}$. We now make an easy remark regarding types over models.

Remark 1.1. Let $\mathscr{K}$ be excellent, $\mathfrak{C} \in \mathscr{K}$ full and $M^{\mathscr{E}} \prec \mathfrak{C}^{\mathscr{E}}$. Then $\operatorname{tp}_{L}(a / M)=$ $\operatorname{tp}_{L}(b / M)$, then $\operatorname{tp}_{L^{\mathscr{E}}}\left(a / M^{\mathscr{E}}\right)=\operatorname{tp}_{L^{\mathscr{E}}}\left(b / M^{\mathscr{E}}\right)$ : This is since there exists an automorphism $f$ of the full model $\mathfrak{C}$, which sends $a$ to $b$. Then $f^{\mathscr{E}}$ is an automorphism of $\mathfrak{C}^{\mathscr{E}}$ fixing $M^{\mathscr{E}}$ sending $a$ to $b$ by Proposition 0.3. Thus, in an excellent class $\mathscr{K}$ the type $\operatorname{tp}(a / M)$ isolates the type $\operatorname{tp}_{L^{\mathscr{E}}}\left(a / M^{\mathscr{E}}\right)$.

Since $\mathscr{K}$ is excellent, then $\mathscr{K}$ has AP, so $\mathscr{K}^{\mathscr{E}}$ also has AP. Recall that $M \in \mathscr{K}$ is $\lambda$-model homogeneous for a cardinal $\lambda$, if whenever $M_{0} \prec N$, with $M_{0} \prec M$ and $\|N\|<\lambda$, there exists an elementary map $g: N \rightarrow M$ which is the identity on $M_{0}$.

Proposition 1.2. $M \in \mathscr{K}$ is $\lambda$-model homogeneous if and only if $M^{\mathscr{E}} \in \mathscr{K}^{\mathscr{E}}$ is $\lambda$-model homogeneous.

Proof. Both directions are similar, so we prove only one. Let $M_{0}^{\mathscr{E}} \prec M^{\mathscr{E}}$, and $M_{0}^{\mathscr{E}} \prec N^{\mathscr{E}}$ with $N^{\mathscr{E}}$ of size less than $\lambda$. Then $M_{0} \prec N$ and by $\lambda$-model homogeneity of $M$ there exists an elementary map $f: N \rightarrow M$ which is the identity on $M_{0}$. Now $f^{\mathscr{E}}: N^{\mathscr{E}} \rightarrow M^{\mathscr{E}}$ is elementary, and since $i d: M_{0}^{\mathscr{E}} \rightarrow N^{\mathscr{E}}$ coincides with $f^{\mathscr{E}}$ on $S_{=}\left(M_{0}^{\mathscr{E}}\right)$, then $f^{\mathscr{E}}$ must be the identity on $M_{0}^{\mathscr{E}}$. Thus $M^{\mathscr{E}}$ is $\lambda$-model homogeneous.

The previous proposition implies that $\mathfrak{C}^{\mathscr{E}}$ is model-homogeneous, and it is unique up to isomophism (by AP).

DEFINITION 1.3. A model is $\lambda$-stable if it realises only $\lambda$ many complete types over subsets of size at most $\lambda$. A class of models $\mathscr{K}$ is $\lambda$-stable if each model is $\lambda$-stable.

If, in addition, $\mathscr{K}$ has AP, then it is easy to see that the following are equivalent:

(1) $\mathscr{K}$ is $\lambda$-stable;

(2) for each $M \in \mathscr{K}$ of size $\lambda$, the following set of types over $M$ has size $\lambda$ :

$$
\{\operatorname{tp}(a / M, N): a \in N, M \prec N \in \mathscr{K}\} .
$$

(3) Every model realises at most $\lambda$ types over elementary submodels of size $\lambda$.

The next proposition uses the countability of $\mathscr{E}$ (though we could get away with defining stability per sort).

Proposition 1.4. $\mathscr{K}^{\mathscr{E}}$ is $\aleph_{0}$-stable.

Proof. Suppose that $M^{\mathscr{E}} \in \mathscr{K}^{\mathscr{E}}$ realises uncountably many types over a countable submodel $N^{\mathscr{E}}$. By taking an elementary extension of $M^{\mathscr{E}}$ if necessary, we may assume that $M$ is uncountable and full (since $\mathscr{K}$ is excellent). Let $\left\{a_{i} / E_{i}\right.$ : $\left.i<\omega_{1}\right\}$ realise distinct types over $N^{\mathscr{E}}$. Since $\mathscr{E}$ is countable, we may assume that $E_{i}=E_{j}=E$ for $i<j$. Then by $\omega$-stability of $M$, there are $i<j$ such that $\operatorname{tp}_{L}\left(a_{i} / N\right)=\operatorname{tp}_{L}\left(a_{j} / N\right)$. Since $M$ is full, there is an automorphism of $M$ fixing $N$ such that $f\left(a_{i}\right)=a_{j}$. Then $f^{\mathscr{E}}$ is an automorphism of $M^{\mathscr{E}}$ fixing $N^{\mathscr{E}}$ (by Proposition 0.2 ) sending $a_{i} / E$ to $a_{j} / E$, a contradiction. 
We now consider the independence relation used to define excellence. It is based on nonsplitting. Recall that $\operatorname{tp}(a / B)$ splits over $A \subseteq B$ if there are $b, c \in B$ with $\operatorname{tp}(b / A)=\operatorname{tp}(c / A)$ but $\operatorname{tp}(a b / A) \neq \operatorname{tp}(a c / A)$ i.e., there is $\phi(x, y) \in L$ with $\phi(x, c), \neg \phi(x, d) \in \operatorname{tp}(a / B)$. Now for sets $A, B$, and $C$, we set

$$
A \underset{B}{\stackrel{n s}{\perp}} C
$$

if and only if for each finite $a \in A$ there exists a finite $B^{\prime} \subseteq B$ such that $\operatorname{tp}(a / B C)$ does not split over $B^{\prime}$. This independence relation is always monotone, invariant, and with finite character, and moreover, when the sets are models, we have symmetry, transitivity, extension, and stationarity. We now consider independent systems:

DeFINITION 1.5. Let $n<\omega$. A system of models $\left(M_{s}: s \subsetneq n\right)$ is an independent $n$-system if

(1) $M_{s} \prec M_{t}$ if $s \subseteq t$.

(2) $M_{s} \underset{M_{s} \cap t}{\stackrel{n s}{\downarrow}} M_{t}$, for each $s, t \subsetneq n$.

Recall that a model $M \in \mathscr{H}$ is primary over a set $A$ if $M=A \cup\left\{a_{i}: i<\alpha\right\}$, where the type of $a_{i}$ over $A \cup\left\{a_{j}: j<i\right\}$ is isolated. Primary models are always prime and it is not difficult to see that, at this level of generality, prime models over countable sets are primary.

We now remind the reader of the definition of excellence in this context.

Definition 1.6. The $\omega$-stable class $\mathscr{K}$ is excellent if for each $n$-independent system of countable models $\left(M_{s}: s \subsetneq n\right), n<\omega$, there exists a primary model over $\bigcup\left\{M_{s}: s \subsetneq n\right\}$.

The next proposition considers 2-dimensional independent systems:

Proposition 1.7. Let $M_{0}^{\mathscr{E}} \prec M_{\ell}^{\mathscr{E}}$ be models. Then

$$
M_{1} \underset{M_{0}}{\stackrel{n s}{\downarrow}} M_{2} \quad \text { if and only if } \quad M_{1}^{\mathscr{E}} \underset{M_{0}^{\mathscr{E}}}{\stackrel{n s}{\downarrow}} M_{2}^{\mathscr{E}} .
$$

Proof. First, let us assume that $M_{1}^{\mathscr{E}} \underset{M_{0}^{\mathscr{E}}}{\stackrel{n s}{\perp}} M_{2}^{\mathscr{E}}$. Let $a \in M_{1}$ be given. Then, there exist $b_{i} \in M_{0}$ for $i<n$ such that $\operatorname{tp}_{L^{\mathscr{E}}}\left(a / M_{2}^{\mathscr{E}}\right)$ does not split over the finite set $b_{0} / E_{0} \ldots b_{n-1} / E_{n-1}$. In particular, $\left.\operatorname{tp}_{L^{\xi}}\left(a / M_{2}^{\mathscr{E}}\right)\right)$ does not split over $b_{0} \ldots b_{n-1}$ : if $c^{\prime}, d^{\prime} \in M_{2}^{\mathscr{E}}$ have the same $L^{\mathscr{E}}$ type over $b_{0} \ldots b_{n-1}$, then they must have the same $L^{\mathscr{E}}$-type over $b_{0} / E_{0} \ldots b_{n-1} / E_{n-1}$. But then $\operatorname{tp}\left(a / M_{2}\right)$ does not split over $b_{0} \ldots b_{n-1}$ by Remark 1.1.

For the converse, assume that $M_{1} \underset{M_{0}}{\stackrel{n s}{\downarrow}} M_{2}$. Let $a_{i} / E_{i} \in M_{1}^{\mathscr{E}}$ for $i<n$. Let $b_{j} \in M_{0}$ for $j<m$ such that $\operatorname{tp}\left(a_{0} \ldots a_{n-1} / M_{2}\right)$ does not split over $b_{0} \ldots b_{m-1}$. We claim that $\operatorname{tp}_{L^{\mathscr{E}}}\left(a_{0} / E_{0} \ldots a_{n-1} / E_{n-1} / M_{2}^{\mathscr{E}}\right)$ does not split over $b_{0} \ldots b_{m-1}$. Otherwise, there are finite sequences $c / E^{\prime}, d / E^{\prime} \in M_{2}^{\mathscr{E}}$ with the same $L^{\mathscr{E}}$-type over $b_{0} \ldots b_{m-1}$ such that the sequences

$$
a_{0} / E_{0} \ldots a_{n-1} / E_{n-1} c / E^{\prime} \text { and } a_{0} / E_{0} \ldots a_{n-1} / E_{n-1} d / E^{\prime}
$$


have distinct $L^{\mathscr{E}}$-type over $b_{0} \ldots b_{m-1}$. We can choose $d^{\prime} \in M_{2}$ so that $M_{2}^{\mathscr{E}} \models$ $d^{\prime} / E^{\prime}=d / E^{\prime}$ and $\operatorname{tp}\left(c b_{0} \ldots b_{m-1}\right)=\operatorname{tp}\left(d^{\prime} b_{0} \ldots b_{m-1}\right)$ (see the proof of Proposition 0.5 ). Thus, the sequences

$$
a_{0} / E_{0} \ldots a_{n-1} / E_{n-1} c / E^{\prime} \text { and } a_{0} / E_{0} \ldots a_{n-1} / E_{n-1} d^{\prime} / E^{\prime}
$$

have distinct $L^{\mathscr{E}}$-types over $b_{0} \ldots b_{m-1}$, but then $\operatorname{tp}\left(a_{0} \ldots a_{n-1} c / b_{0} \ldots b_{m-1}\right) \neq$ $\operatorname{tp}\left(a_{0} \ldots a_{n-1} d^{\prime} / b_{0} \ldots b_{m-1}\right)$, a contradiction.

From this, the next proposition follows immediately:

Proposition 1.8. Let $\left(M_{s}^{\mathscr{E}}: s \subsetneq n\right)$ be such that $M_{s}^{\mathscr{E}} \prec M_{t}^{\mathscr{E}}$ for $s \subseteq t$. Then $\left(M_{s}^{\mathscr{E}}: s \subsetneq n\right)$ is an independent $n$-system if and only if $\left(M_{s}: s \subsetneq n\right)$ is an independent n-system.

We write that $a \in b c l(A)$ (bounded closure) if the number of realizations in the monster model $\mathfrak{C}$ of $\operatorname{tp}(a / A)$ is bounded i.e., less than the size of the monster model. We write that $a \in \operatorname{dcl}(A)$ (definable closure) if there is a formula $\phi$ and $b \in A$ such that $a$ is the only realization of $\phi(x, b)$ in the monster model. We write $b c l^{\mathscr{E}}$ and $d c l^{\mathscr{E}}$ when the closures are taken in $\mathfrak{C}^{\mathscr{E}}$. Notice that $M^{\mathscr{E}}=d c l^{\mathscr{E}}(M)$.

Now we have almost shown that if $\mathscr{K}$ is excellent then so is $\mathscr{K}^{\mathscr{E}}$. Only the following simple observation is needed: Suppose $A \subseteq \mathfrak{C}, B \subseteq d c l^{\mathscr{E}}(A)$ is such that $A \subseteq B$ and $M \subseteq \mathfrak{C}$ is primary over $A$. Then $M^{\mathscr{E}}$ is primary over $B$ (clearly $M$ is constructible over $B, M^{\mathscr{E}}$ is constructible over $M B$ and $M^{\mathscr{E}}$ is an elementary submodel of $\mathfrak{C}^{\mathscr{E}}$ and so also $B \subseteq M^{\mathscr{E}}$ ).

THEOREM 1.9. If $\mathscr{K}$ is excellent then $\mathscr{K}^{\mathscr{E}}$ is excellent. Furthermore $M \in \mathscr{K}$ is full if and only if $M^{\mathscr{E}} \in \mathscr{K}^{\mathscr{E}}$ is full.

PRoOF. We must show that for any $n$ and for any independent $n$-system $\left(M_{s}^{\mathscr{E}}\right.$ : $s \subsetneq n)$ of countable models, there exists $M^{\mathscr{E}}$ primary over $\bigcup_{s \subsetneq n} M_{s}^{\mathscr{E}}$.

So let $\left(M_{s}^{\mathscr{E}}: s \subsetneq n\right)$ be an $n$-independent system of countable models. Then $\left(M_{s}: s \subsetneq n\right)$ is an $n$-independent system of countable models so by excellence of $\mathscr{K}$ there exists $M$ primary over $\bigcup_{s \subsetneq n} M_{s}$. By the observation above, $M^{\mathscr{E}}$ is as wanted.

The furthermore part follows from Proposition 1.2 (it is easy to see under the definition of excellence that uncountable full models and uncountable model homogeneous models coincide and so if $M$ is model homogeneous then so is $M^{\mathscr{E}}$ by Proposition 1.2).

In order to consider simplicity, we have to look at a different independence relation, based on Lascar splitting. For this, we examine the behaviour of indiscernibles.

Definition 1.10. We say that $\left(a_{i} / E: i<\omega\right) \subseteq \mathfrak{C}^{\mathscr{E}}$ is strongly indiscernible over $A$ if for each $\lambda$, we can find $\left(a_{i} / E: \omega \leq i<\lambda\right)$ with the property that any permutation of $\left(a_{i} / E: i<\lambda\right)$ extends to an automorphism of $\mathfrak{C}^{\mathscr{E}}$ fixing $A$.

The next lemma is the key reason why independence is well-behaved here.

LEMMA 1.11. Let $\left(a_{n} / E: n<\omega\right) \subseteq \mathfrak{C}^{+\mathscr{E}}$ be strongly indiscernible over the finite set $b_{0} / E_{0} \ldots b_{m-1} / E_{n-1}$. Then there exist $\left(a_{n}^{\prime}: n<\omega\right) \subseteq \mathfrak{C}$ and $b_{i}^{\prime} \in \mathfrak{C}$ for $i<m$ such that $b_{i} / E_{i}=b_{i}^{\prime} / E_{i}$ for $i<m$ and $\left(a_{n}^{\prime}: n<\omega\right)$ is strongly indiscernible over $b_{0}^{\prime} \ldots b_{n-1}^{\prime}$.

Proof. By strong indiscernibility of $\left(a_{n} / E: n<\omega\right)$ in $\mathfrak{C}^{\mathscr{E}}$, we can find $\left(a_{i} / E\right.$ : $i<\lambda)$ extending $\left(a_{n} / E: n<\omega\right)$ strongly indiscernible over $b_{0} / E_{0} \ldots b_{n-1} / E_{n-1}$. 
Then by $\omega$-stability and excellence of $\mathfrak{C}$, we can find $\left(a_{i_{n}}: n<\omega\right)$ strongly indiscernible in $\mathfrak{C}$ over $b_{0} \ldots b_{n-1}$. By strong indiscernibility of $\left(a_{i} / E: i<\lambda\right)$ over $b_{0} / E_{0} \ldots b_{n-1} / E_{n-1}$, there is an automorphism $f^{\mathscr{E}}$ of $\mathfrak{C}^{\mathscr{E}}$ fixing $b_{i} / E_{i}, i<m$. such that $f^{\mathscr{E}}\left(a_{i_{n}} / E\right)=a_{n} / E$, for each $n<\omega$. Let $a_{n}^{\prime}=f^{\mathscr{E}}\left(a_{i_{n}}\right)$ and $b_{i}^{\prime}:=f^{\mathscr{E}}\left(b_{i}\right)$. These are as desired.

The next definition was introduced in [HyLe] at this level of generality.

Definition 1.12. We say that $\operatorname{tp}(a / A)$ Lascar-splits over $B \subseteq A$ if there is a strongly $B$-indiscernible sequence $\left(a_{i}: i<\omega\right)$ with $a_{0}, a_{1} \in A$ such that

$$
\operatorname{tp}\left(a a_{0} / B\right) \neq \operatorname{tp}\left(a a_{1} / B\right) .
$$

Proposition 1.13. Let $A \subseteq M$ finite and $A^{\prime} \subseteq M^{\mathscr{E}}$ such that dcl ${ }^{\mathscr{E}}(A)=$ $d c l^{\mathscr{E}}\left(A^{\prime}\right)$. Then $\operatorname{tp}(a / M)$ Lascar-splits over $A$ if $\operatorname{tp}_{L^{\mathscr{E}}}\left(a / E / M^{\mathscr{E}}\right)$ Lascar-splits over $A^{\prime}$.

Proor. So suppose that $\operatorname{tp}_{L^{\mathscr{E}}}\left(a / E / M^{\mathscr{E}}\right)$ Lascar-splits over $A^{\prime}$. Let $\left(b_{i} / F: i<\omega\right)$ be strongly indiscernible over $A^{\prime}$ with $b_{0} / F, b_{1} / F \in M^{\mathscr{E}}$ and $\operatorname{tp}_{L^{\mathscr{E}}}\left(a / E, b_{0} / F / A^{\prime}\right) \neq$ $\operatorname{tp}\left(a / E, b_{1} / F / A^{\prime}\right)$. By the previous lemma (and atomicity of $M^{\mathscr{E}}$ ), we may assume that $\left(b_{i}: i<\omega\right)$ are strongly $A$-indiscernible (in $\left.M\right)$. Since $\operatorname{tp}_{L}\left(a b_{0} / A\right) \neq$ $\operatorname{tp}\left(a b_{1} / A\right)$, we are done.

We write $a \underset{A}{\downarrow} B$ and say that $\operatorname{tp}(a / A B)$ is a free extension of $\operatorname{tp}(a / A)$ if there is finite $C \subseteq A$ such that for all $D \supseteq A B$ there is $b$ realizing $\operatorname{tp}(a / A B)$ such that $\operatorname{tp}(b / D)$ does not Lascar-split over $C$. We write $A \underset{B}{\downarrow} C$ if for all (finite) $a \in A$, $a \underset{B}{\downarrow} C$. For models $M$ and finite $A \subseteq M, a \underset{A}{\downarrow} M$ if and only if $\operatorname{tp}(a / M)$ does not Lascar-split over $A$, see [HyLe].

Suppose that $M_{0}^{\mathscr{E}} \prec M_{\ell}^{\mathscr{E}}$ for $\ell=1,2$. Then the previous proposition shows that $M_{1}^{\mathscr{E}} \underset{M_{0}^{\mathscr{E}}}{\downarrow} M_{2}^{\mathscr{E}}$ if and only if $M_{1} \underset{M_{0}}{\downarrow} M_{2}$.

We now show that simplicity follows from finiteness of $U$-rank. Simplicity means that the independence notion $\downarrow$ has all the usual properties of non-forking. In

excellent classes, $U$-rank can be defined as in the elementary case for types over models. Then we say that $\mathscr{H}$ is of finite $U$-rank if for all $a \in \mathfrak{C}$, there is $n<\omega$ such that for all $M \subseteq \mathfrak{C}$, the $U$-rank of $\operatorname{tp}(a / M)$ is $<n$ (whether $a$ is a singleton or a finite tuple is irrelevant in this definition). The main result of [HyLe] is that if $\mathscr{K}$ has finite $U$-rank, then it must be simple. For more on simplicity and $U$-rank, see [Lel] and [HyLe].

THEOREM 1.14. If $\mathscr{K}$ is excellent and of finite U-rank then $\mathscr{K}^{\mathscr{E}}$ is excellent and simple.

Proof. Since we have already shown that $\mathscr{K}^{\mathscr{E}}$ is excellent, by the result of [HyLe], it is enough to show that $\mathscr{K}^{\mathscr{E}}$ is of finite $U$-rank. This is clear by the previous proposition.

One can prove this theorem also essentially as the related result was proved in [Hy] or [HyLe]. From that proof, a weaker property than finiteness of $U$-rank implies the simplicity of $\mathscr{K}^{\mathscr{E}}$, but that property is still stronger than simplicity of $\mathscr{K}$ is simple. It is not clear to us, whether simplicity of $\mathscr{K}$ alone implies the simplicity of $\mathscr{K}^{\mathscr{E}}$. 
We finish this section with a corollary which provides further evidence that excellent classes are natural, appearing as the closure of homogeneous classes.

COROLLARY 1.15. Let $\mathscr{K}$ be a homogeneous $\omega$-stable atomic class of models. Then $\mathscr{K}^{\mathscr{\mathscr { C }}}$ is $\omega$-stable and excellent. If $\mathscr{K}$ is, in addition, of finite $U$-rank, then $\mathscr{K}^{\mathscr{E}}$ is excellent and simple.

Proof. This follows immediately from the fact that $\mathscr{K}$ must be excellent.

§2. Expanding with canonical bases; the general case. In this section, we fix an excellent atomic class $\mathscr{K}$ and work inside a large full model $\mathfrak{C} \in \mathscr{K}$. We will consider nonsplitting and types over models, and in particular global types which are those types over $\mathfrak{C}$.

Global types have the following immediate property with respect to nonsplitting. The proof is clear from the definitions and the strong $\omega$-homogeneity of $\mathfrak{C}$.

Proposition 2.1. Let $p \in \mathrm{S}_{\mathrm{at}}(\mathfrak{C})$ and let $C \subseteq \mathfrak{C}$ be finite. The following conditions are equivalent:

(1) $f(p)=p$ for each $f \in A u t(\mathbb{C} / C)$.

(2) $p$ does not split over $C$.

Furthermore, (2) implies (1) also without the assumption that $C$ is finite.

We now consider type-definability of types over models:

DEFINITION 2.2. We say that $p \in \mathrm{S}_{\mathrm{at}}(M)$ is type definable over $C \subseteq M$ if for each $\phi(x, y)$ there exists a type $d_{\phi}^{p}(y, c)$ over $C$ such that for each $b \in M$ we have

$$
\phi(x, b) \in p \quad \text { if and only if } b \models d_{\phi}^{p}(y, c) .
$$

We call the type $d_{\phi}^{p}(x, c)$ the definition of $\phi$ in $p$ over $c$. It is clear that the definition is (semantically) unique.

Proposition 2.3. Let $p \in \mathrm{S}_{\mathrm{at}}(M)$ and $C \subseteq M$ be finite. Then the following conditions are equivalent:

(1) $p$ does not split over $C$.

(2) $p$ is type-definable over $C$.

Proof. (2) implies (1) is clear, so let us prove (1) implies (2): Assume that $p$ does not split over $C$. Let $c$ be an enumeration of $C$. Let $\bar{p} \in \mathrm{S}_{\mathrm{at}}(\mathfrak{C})$ be the unique nonsplitting (over $c$ ) extension of $p$. It is enough to show that $\bar{p}$ is type-definable over $c$. For $\phi(x, b)$ and $b \in \mathfrak{C}$, then $\phi(x, b) \in \bar{p}$ if and only if $\phi(x, f(b)) \in \bar{p}$ for any automorphism $f$ of $\mathfrak{C}$ fixing $c$ (this is simply by nonsplitting). Thus, this property is invariant over $c$. By strong $\omega$-homogeneity and atomicity of $\mathfrak{C}$, this implies immediately that there is a type $d_{\phi}^{\bar{p}}(y, c)$ over $c$ such that $b \models d_{\phi}(y)^{\bar{p}}$ if and only if $\phi(x, b) \in \bar{p}$ (see the observation before Definition 0.1 ). This shows that $\bar{p}$ (hence $p$ ) is type-definable over $c$.

Now, each $p \in \mathrm{S}_{\mathrm{at}}(M)$ does not split over a finite set $c$, and is therefore typedefinable over $c$. This allows us to define an invariant equivalence relation

$$
E^{p}\left(c, c^{\prime}\right)
$$

to hold if for any $b \in \mathfrak{C}$ and for any $\phi(x, y)$,

$$
b \models d_{\phi}^{p}(y, c) \text { if and only if } b \models d_{\phi}^{p}\left(y, c^{\prime}\right) .
$$


Definition 2.4. Let $\mathscr{E}(\mathscr{K}) \subseteq T E(\mathscr{K})$ be the closure of $\left\{E^{p}: p \in \mathrm{S}_{\mathrm{at}}(M)\right\}$, where $M \in \mathscr{K}$ is the unique countable model, under all first order operations.

Notice that $\mathscr{E}(\mathscr{K})$ does not depend on $M$ since any two countable submodels of $\mathfrak{C}$ are automorphic (since $\mathscr{K}$ is atomic, countable models are full and thus isomorphic, even over any finite common part). Furthermore $\mathscr{E}(\mathscr{K})$ is countable.

Theorem 2.5. Let $\mathscr{E}=\mathscr{E}(\mathscr{K})$. For each $p \in \mathrm{S}_{\mathrm{at}}(\mathfrak{C})$ there exists an element $C b(p) \in \mathfrak{C}^{\mathscr{E}}$ such that

$$
\begin{gathered}
f(p)=p \text { if and only if } f(C b(p))=C b(p), \\
\text { for any automorphism } f \text { of } \mathfrak{C}^{\mathscr{E}} .
\end{gathered}
$$

Furthermore, $C b(p)$ is unique up to interdefinability $\left(\right.$ in $\left.L^{\mathscr{E}}\right)$.

Proof. Let $p \in \mathrm{S}_{\mathrm{at}}(\mathfrak{C})$ be given and choose $c \subseteq \mathfrak{C}$ such that $p$ does not split over $c$. Let $N$ be a countable model containing $c$. Then $N$ is automorphic to $M$ over $c$. Hence, we may assume that $N=M$ (in the definition of $\mathscr{E}$ ). Let $E^{p} \in \mathscr{E}$ associated with $p\left\lceil M\right.$. Let $C b(p):=c / E^{p}$.

Let $f$ be an automorphism of $\mathcal{C}^{\mathscr{E}}$ such that $f(p)=p$. To show that $f(C b(p))=$ $C b(p)$, it is enough to show that $E^{p}(c, f(c))$. Let $\phi(x, b)$ be given. Then $\phi(x, b) \in$ $p$ if and only if $b \models d_{\dot{\phi}}^{p}(y, c)$. But $p=f(p)$ so $\phi(x, b) \in p$ if and only if $\phi(x, b) \in f(p)$ if and only if $b \models d_{\phi}^{p}(y, f(c))$. Thus $E^{p}(c, f(c))$.

Now suppose that $f$ is an automorphism of $\mathfrak{C}^{\mathscr{E}}$ such that $E^{p}(c, f(c))$. Then $\phi(x, b) \in p$ if and only if $b \models d_{\phi}^{p}(y, c)$ if and only if $b \models d_{\phi}^{p}(y, f(c))$ (since $\left.E^{p}(c, f(c))\right)$ if and only if $f^{-1}(b) \neq=d_{\phi}^{p}(y, c)$ if and only if $\phi\left(x, f^{-1}(b)\right) \in p$ if and only if $\phi(x, b) \in f(p)$. Thus $p=f(p)$.

For the last sentence, if $d \in \mathbb{C}^{\mathscr{E}}$ is such that $f(d)=d$ if and only if $f(p)=p$ for any $f \in A u t\left(\mathbb{C}^{\mathscr{E}}\right)$, then $f(d)=d$ if and only if $f(C b(p))=C b(p)$. This implies that $d$ is the only realisation of the isolated type $\operatorname{tp}_{L^{f}}(d / C b(p))$ and $C b(p)$ the only realisation of the isolated type $\operatorname{tp}_{L^{\xi}}(C b(p) / d)$. Thus, $d$ and $C b(p)$ are interdefinable in $L^{\mathscr{E}}$.

Definition 2.6. Let $\mathscr{K}$ be an excellent class, and $p \in \mathrm{S}_{\mathrm{at}}(\mathfrak{C})$. We call an element $c \in \mathfrak{C}$ the canonical basis for $p$ if $c$ has the property that

$$
f(c)=c \text { if and only if } f(p)=p, \quad \text { for each automorphism } f \text { of } \mathfrak{C} .
$$

We say that $\mathscr{K}$ has canonical bases if each $p \in \mathrm{S}_{\mathrm{at}}(\mathfrak{C})$ has a canonical basis.

The previous theorem does not quite show that $\mathfrak{C}^{\mathscr{E}}$ has canonical bases, as we have provided one only for global types in the equality sort. However, remedying this is easy; we simply iterate the process $\omega$-times: Let $\mathscr{E}_{0}=\mathscr{E}\left(\mathscr{K}_{0}\right)$. We let $\mathscr{K}_{1}=\left(\mathscr{K}_{0}\right)^{\mathscr{E}_{0}}$ and call $M_{1} \in \mathscr{K}_{1}$ the expansion of $M_{0}$. By work done so far, $\mathscr{K}_{1}$ is an atomic, excellent class in a countable language. Inductively, having constructed an atomic, excellent class in a countable language $L_{n}$ and $M_{n}$ an expansion of $M$ in this language (unique up to isomorphism), we let $\mathscr{K}_{n+1}=\left(\mathscr{K}_{n}\right)^{\mathscr{E}_{n}}$, where $\mathscr{E}_{n}=\mathscr{E}\left(\mathscr{K}_{n}\right)$ computed with $M_{n}$, and let $M_{n+1}=M_{n}^{\mathscr{E}_{n}}$. We consider $L^{e q}$ the union of all $L_{n}$ and for each $N \in \mathscr{K}$ we let $N^{e q}$ be the expansion of $N$ in $L^{e q}$ which extends all $L_{n}$ (this is unambiguous). We make a remark familiar from the elementary case. 
RemarK 2.7. Let us look at the second step in the iteration: Suppose $E \in \mathscr{E}_{1}$ is such that on $M^{\mathscr{E}_{0}} \in \mathscr{K}_{1}, E$ is a subset of $S_{E_{0}}\left(M^{\mathscr{E}_{0}}\right) \times \cdots \times S_{E_{n}}\left(M^{\mathscr{E}_{0}}\right)$. Let $E^{*} \in T E(\mathscr{K})$ be such that

$$
E^{*}\left(\left(a_{0}, \ldots, a_{n}\right),\left(b_{0}, \ldots, b_{n}\right)\right)
$$

holds iff

$$
E\left(\left(a_{0} / E_{0}, \ldots, a_{n} / E_{n}\right),\left(b_{0} / E_{0}, \ldots, b_{n} / E_{n}\right)\right)
$$

holds. Then if we identify the class $\left(a_{0}, \ldots, a_{n}\right) / E^{*}$ with $\left(a_{0} / E_{0}, \ldots, a_{n} / E_{n}\right) / E$, we see that $\left(M^{\mathscr{E}_{0}}\right)\{E\}$ is essentially the same structure as $M^{\mathscr{E}_{0} \cup\left\{E^{*}\right\}}$, everything that is type definable in one is also type definable in the other. Thus we will not distinguish $E$ and $E^{*}$ and in the inductive process above, $\mathscr{E}_{n}$ simply corresponds to a larger subset $\mathscr{E}_{n}^{\prime} \subseteq T E(\mathscr{K})$, so that we could have achieved the expansion $\mathscr{K}^{e q}$ with one subset $E^{\prime} \subseteq T E(\mathscr{K})$, namely $\bigcup_{n<\omega} \mathscr{E}_{n}$.

THeOREM 2.8. Let $\mathscr{K}$ be an excellent atomic class in the countable language $L$. Then $\mathscr{K}^{e q}$ is excellent, atomic, and has canonical bases.

PRoof. The key point is that any type $p \in \mathrm{S}_{\mathrm{at}}\left(\mathfrak{C}^{e q}\right)$ in finitely many variables, is the only extension of a type $p_{n}$ in $\mathrm{S}_{\mathrm{at}}\left(\mathfrak{C}^{\mathscr{E}_{n}}\right)$ which follows from Remark 1.1. Then, the canonical basis for $p_{n}$ (and hence $p$ ) exists in $\mathfrak{C}^{\mathscr{E}_{n+1}}$, hence in $\mathfrak{C}^{e q}$.

We finish this section with an easy observation, which we need in the next section.

Proposition 2.9. Suppose $\mathscr{K}$ is an excellent atomic class in the countable language $L$ and let $\mathscr{E}=\mathscr{E}(\mathscr{K})$. Let $p \in \mathrm{S}_{\mathrm{at}}(\mathfrak{C})$ and let $q \in \mathrm{S}_{\mathrm{at}}\left(\mathfrak{C}^{\mathscr{E}}\right)$ be the unique extension of p. Then $q$ does not split over a finite set $A \subseteq \mathfrak{C}^{\mathscr{E}}$ if and only if $C b(p) \in d c l^{\mathscr{E}}(A)$. In particular, if $p \in \mathrm{S}_{\mathrm{at}}\left(\mathfrak{C}^{e q}\right)$ then $p$ does not split over a finite set $A \subseteq \mathfrak{C}^{\text {eq }}$ if and only if $C b(p) \in d c l^{e q}(A)$.

Proof. Since $q$ does not split over a finite set $A$ if and only if $f(q)=q$ for any automorphism $f$ of $\mathfrak{C}^{\mathscr{E}}$ fixing $A$ and since $q$ is the unique extension of $p, q$ does not split over a finite set $A$ if and only if $f(p)=p$ for any automorphism $f$ of $\mathfrak{C}^{\mathscr{E}}$ fixing $A$. So if $C b(p) \in d c l^{\mathscr{E}}(A), q$ does not split over $A$.

For the converse, if $q$ does not split over finite $A$, then any automorphism of $\mathfrak{C}^{\mathscr{E}}$ fixing $A$ fixes $q$ and thus $p$ and thus fixes $C b(p)$. This implies that $C b(p)$ is the only element realising $\operatorname{tp}(C b(p) / A)$, so $C b(p) \in d c l^{\mathscr{E}}(A)$.

The in particular part follows as in the proof of the previous theorem.

§3. Expanding with canonical bases; the case of finite $U$-rank. Assume that $\mathscr{K}$ is excellent and of finite $U$-rank. Then we have an independence relation which satisfies all the usual properties of nonforking, and for which Lascar strong types are stationary, see [HyLe]. In order to obtain a better Canonical Basis Theorem, we will expand $L$, not only with $E^{p}$, but also with equivalence relations capturing equality between Lascar strong types.

Let us consider Lascar strong types over finite sets: Notice that

$$
\operatorname{Lstp}(a / c)=\operatorname{Lstp}(b / c)
$$

if and only if $\operatorname{Lstp}(f(a) / f(c))=\operatorname{Lstp}(f(b) / f(c))$. Also, since equality between Lascar strong types of $n$-tuples over the finite set $c$ is $c$-invariant, then there exists a type $E(x, y ; c)$ over $c$ representing it in $\mathfrak{C}$. Notice also that if $\operatorname{tp}\left(c^{\prime} / \emptyset\right)=\operatorname{tp}(c / \emptyset)$, then $E\left(x, y ; c^{\prime}\right)$ represents equality of Lascar strong types of $n$-tuples over $c^{\prime}$. 
Finally, if $E(x, y ; c)$ is a $c$-invariant equivalence relation, we can form the invariant equivalence relation $E^{\prime}\left(x, z_{1} ; y, z_{2}\right)$ defined by $E^{\prime}\left(a_{1} b_{1} ; a_{2} b_{2}\right)$ if $b_{1}=b_{2}$ and either $b_{1} \not \neq \operatorname{tp}(c / \emptyset)$ or $E\left(a_{1}, a_{2} ; b_{1}\right)$. Then $E^{\prime}$ is type-definable over the empty set and $E(a, b ; c)$ if and only if $E^{\prime}(a c ; b c)$.

This motivates the following definition:

DEFINITION 3.1. We let $\mathscr{E}^{*}(\mathscr{K}) \subseteq T E(\mathscr{K})$ be the closure under first order operations of $\mathscr{E}(\mathscr{K})$ and equivalence relations $E^{\prime}\left(x, z_{1} ; y, z_{2}\right)$, where $E(a, b ; c)$ is equality of Lascar strong types over $c$.

It follows from the discussion preceding the definition that $\mathscr{E}^{*}(\mathscr{K})$ is countable. The main point achieved is the following theorem:

TheOREM 3.2. Let $\mathscr{E}^{*}=\mathscr{E}^{*}(\mathscr{K})$ and $a \in \mathfrak{C}$. The types $\operatorname{tp}_{L^{\mathscr{F}^{*}}}\left(a / b c l^{\mathscr{E}^{*}}(C)\right)$ are stationary.

PROOF. Without loss of generality (by simplicity), we may assume that $C$ is finite, which we write $c$. Since Lascar strong types over $c$ are stationary, it is enough to show that if $b \models \operatorname{tp}_{L^{z^{*}}}\left(a / b c l^{\mathscr{\ell}^{*}}(c)\right)$, then $\operatorname{Lstp}(a / c)=\operatorname{Lstp}(b / c)$. Let $E^{\prime}\left(x, z_{1} ; y, z_{2}\right) \in$ $\mathscr{E}^{*}$ be such that $\operatorname{Lstp}(a / c)=\operatorname{Lstp}(d / c)$ if and only if $E^{\prime}(a c ; b c)$. Then, the type $\operatorname{tp}_{L^{\mathscr{E}^{*}}}\left(\left(a c / E^{\prime}\right) / c\right)$ is bounded by definition of Lascar strong types, so the element $a c / E^{\prime} \in b c l^{\mathscr{F}^{*}}(c)$, so the formula $F_{E}^{\prime}(x, c)=a c / E^{\prime}$ belongs to $\operatorname{tp}_{L^{\mathscr{E}}}\left(a / b c l^{\mathscr{E}^{*}}(c)\right)$, and so $b c / E^{\prime}=a c / E^{\prime}$, which implies that $\operatorname{Lstp}(a / c)=\operatorname{Lstp}(b / c)$, which is what we wanted.

Suppose that $p \in \mathrm{S}_{\mathrm{at}}(\mathfrak{C})$ is a free extension of $p\lceil A$ for some finite $A \subseteq \mathfrak{C}$. By $p$ we denote also the unique extension of $p$ over $\mathfrak{C}^{\mathscr{C}^{*}}$ We write $C b^{*}(p, A)$ for $a A / E^{\prime}$, where $E^{\prime}$ is as above and $a \models p \nmid M$ for some model $A \subseteq M \subseteq \mathfrak{C}$. Notice that this does not depend on the choice of $a$ or $M$ and that $C b^{*}(p, A) \in b c l^{\mathscr{E}^{*}}(A)$. From the proof of the previous theorem, it follows that $p \nmid A \cup\left\{C b^{*}(p, A)\right\}$ is stationary. Notice also that $C b(p)$ (as defined in the previous section) and $C b^{*}(p, A)$ are interdefinable over $A$ : If $f$ is an automorphism of $\mathfrak{C}^{\mathscr{E}^{*}}$ and it fixes $A \cup\left\{C b^{*}(p, A)\right\}$, then by stationarity, it fixes $p$. Thus it fixes $C b(p)$, and so $C b(p) \in d c l^{\mathscr{E}^{*}}\left(A \cup\left\{C b^{*}(p, A)\right\}\right)$. For the other direction, suppose that there is an automorphism $f$ of $\mathfrak{C}^{\mathscr{E}^{*}}$ such that it fixes $A$ and $C b(p)$ but moves $C b^{*}(p, A)$. Let $a$ and $M$ be as in the definbition of $C b^{*}(p, A)$ and let $b$ realize $f(p) \uparrow M$. Since now $\operatorname{Lstp}(b / A) \neq \operatorname{Lstp}(b / A), \operatorname{tp}(b / M) \neq \operatorname{tp}(a / M)$ and thus $f(p) \neq p$ which contradicts the fact that $f(C b(p))=C b(p)$.

TheOREM 3.3. Let $p \in \mathrm{S}_{\mathrm{at}}(\mathfrak{C})$. Then $p$ has a canonical basis $C b(p) \in \mathfrak{C}^{\mathscr{E}^{*}}$. Furthermore:

(1) $p$ is a free extension of $p \nmid A$ if and only if $C b(p) \in b c l^{\mathscr{E}^{*}}(A)$.

(2) if $C b(p) \in d c l^{\mathscr{E}^{*}}(A)$ then $p$ is a free extension of $p\lceil A$ and $p\lceil A$ is stationary and the converse holds if $A$ is finite.

Proof. First, $p$ extends uniquely to $S_{\text {at }}\left(\mathfrak{C}^{\mathscr{E}}\right)$, so we may assume that $p$ is in fact in $S_{a t}\left(C^{\mathscr{E}^{*}}\right)$. (1): Assume first that $p$ is a free extension of $p \nmid A$, then $p$ is a free extension of $p\lceil C$ for some finite $C \subseteq A$. By the observation above, $C b(p) \in$ $b c l^{\mathscr{E}^{*}}(A)\left(C b^{*}(p, C) \in b c l^{\mathscr{E}^{*}}(C)\right.$ and $\left.C b(p) \in d c l^{\mathscr{E}^{*}}\left(C \cup\left\{C b^{*}(p, C)\right\}\right)\right)$.

For the converse, if $C b(p) \in b c l^{\mathscr{E}^{*}}(A)$, then $C b(p) \in b c l^{\mathscr{E}^{*}}(C)$ for some finite $C \subseteq A$ (see [HyLe]). Since $p$ does not split over $C b(p)$, there are only boundedly 
many types to which automorphisms of $\mathfrak{C}^{\mathscr{E} *}$ which fix $C$ can move $p$. So $p$ does not Lascar split over $C$.

(2): Suppose that $C b(p) \in d c l^{\mathscr{E} *}(A)$. By (1), $p$ is a free extension of $p \mid A$. We need to show that $p\lceil A$ is stationary. For this let $B \subseteq A$ be finite such that $p$ is a free extension of $p \nmid B$. Then by the observation above, $C b^{*}(p, B) \in d c l^{\mathscr{E}^{*}}(A)$ and so there is only one extension of $p\left\lceil A\right.$ to a type over $A \cup\left\{C b^{*}(p, B)\right\}$. Since $p\left\lceil A \cup\left\{C b^{*}(p, B)\right\}\right.$ is stationary, also $p\lceil A$ must be stationary.

For the converse, assume that $p$ is a free extension of $p\lceil A, p\lceil A$ is stationary and $A$ is finite. Then $f(p)=p$ for all automorphisms $f$ of $\mathcal{C}^{\mathscr{E}^{*}}$ that fix $A$. So by the definition of canonnical bases, $f(C b(p))=C b(p)$ for all such automorphisms and thus $C b(p) \in d c l^{\mathscr{E}^{*}}(A)$.

We point out that in (2), finiteness of $A$ was needed because $d c l$ may behave unexpectedly in excellent classes, i.e., $a$ may not be in $\operatorname{dcl}(A)$ even if $f(a)=a$ for all automorphisms that fix $A$. If $A$ is finite, this can not happen.

By expanding $\omega$-times, as in the end of the previous section, but closing under $\mathscr{E}^{*}(\mathscr{K})$, instead of $\mathscr{E}(\mathscr{K})$, we can obtain an atomic, excellent, simple class with canonical bases and such that types over the bounded closure of sets are all stationary. We call the resulting expanded language $L^{e q}$ again (it can also be obtained in one step) and the resulting class $\mathscr{K}^{e q}$. We then have:

THEOREM 3.4 (Canonical Basis Theorem). Let $\mathscr{K}$ be an excellent, atomic class in a countable language and of finite $U$-rank. Then there exists a simple, excellent, atomic expansion $\mathscr{K}^{e q}$, in a countable language, which has canonical bases such that:

(1) For any $a$ and $C$ in $\mathfrak{C}^{e q}$, the type $\operatorname{tp}_{L^{e q}}\left(a / b c l^{e q}(C)\right)$ is stationary.

(2) If $p \in \mathrm{S}_{\mathrm{at}}(\mathfrak{C})$ then the canonical basis $C b(p)$ has the following properties:

(a) $p$ is free over $C$ if and only if $C b(p) \in b c l^{e q}(C)$;

(b) if $C$ is finite, then $p$ is free over $C$ and $p \nmid C$ is stationary if and only if $C b(p) \in d c l^{e q}(C)$ if and only if $p$ is definable over $C$ if and only if $p$ does not split over $C$.

Proof. As before, types in $\mathrm{S}_{\mathrm{at}}\left(\mathfrak{C}^{e q}\right)$ are determined at a finite stage of the construction. So just repeat the proof of Theorem 3.3.

\section{REFERENCES}

[BY] ITAY BEN YAACOV, Discouraging results with ultra-imaginaries, preprint.

[HKP] BRadd HaRT, Byunghan KIM, and ANand Pillay, Coordinatisation and canonical bases in simple theories, this JourNal, vol. 65 (2000), no. 1, pp. 293-309.

[Hy] Tapani HytTinen, Finiteness of $U$-rank implies simplicity in homogeneous structures, Mathematical Logic Quaterly, vol. 49 (2003), no. 6, pp. 576-578.

[HyLe] TAPANI HYTtINEN and OLIVIER LESSMANN, Simplicity and uncountable categoricity in excellent classes, Annals of Pure and Applied Logic, vol. 139 (2006), no. 1-3, pp. 110-137.

[HLS] TAPANI HYTTINEN, OLIVIER LESSMANN, and SAHARON SHELAH, Interpreting groups and fields in some nonelementary classes, Journal of Mathematical Logic, vol. 5 (2005), no. 1, pp. 1-47.

[Le1] Olivier Lessmann, Categoricity and U-rank in excellent classes, this Journal, vol. 68 (2003), no. 4, pp. 1317-1336.

[Le2] - An introduction to excellent classes, Logic and its applications, Contemporary Mathematics, vol. 380, AMS, Providence, RI, 231-259 2005.

[Sh48] SaHARON SHELAH, Categoricity in $\aleph_{1}$ of sentences in $L_{\omega_{1} \omega}(Q)$, Israel Journal of Mathematics, vol, 20 (1975), pp. 127-148. 
[Sh87a] - Classification theory for nonelementary classes. I. The number of uncountable models of $\psi \in L_{\omega_{1}{ }^{\prime}}$. Part A, Israel Journal of Mathematics, vol. 46 (1983), pp. 212-240.

[Sh87b] - Classification theory for nonelementary classes. 1 . The number of uncountable models of $\psi \in L_{\ell_{1}(3 .}$. Part B, Israel Journal of Mathematics, vol. 46 (1983), pp. 241-273.

[Zi] BorIs ZILBER, Pseudo-exponentiation on algebraically closed fields of characteristic zero, Annals of Pure and Applied Logic, vol. 132 (2005), no. 1, pp. 67-95.

DEPARTMENT OF MATHEMATICS AND STATISTICS

P.O. BOX 4

00014 UNIVERSITY OF HELSINKI. FINLAND

E-mail: tapani.hyttinen@helsinki.fi

COLLÉGE ROUSSEAU

CASE POSTALE 216

1211 GENEVA 28, SWITZERLAND

E-mail: lessmann@gmail.com 